\title{
Current status of SciCRT experiment and its expected future performance
}

\author{
Marcos Anzorena $^{* a}$, J.F.Valdés-Galicia ${ }^{a}$, Rocío García $^{a}$, Yutaka Matsubara $^{b}$, \\ Yoshinori Sasai $^{b}$, Tetsuya Kawabata ${ }^{b}$, Ernesto Ortiz $^{c}$, L.X.González ${ }^{d}$, Octavio \\ Musalem $^{a}$, Alejandro Hurtado ${ }^{a}$, Marco Barrantes $^{a}$, Roberto Taylor $^{a}$, Yoshitaka Itow $^{b}$, \\ Takashi Sako $^{b}$, Akira Tsuchiya $^{b}$, Kazuoki Munakata $^{e}$, Chihiro Kato $^{e}$, Yoshiaki \\ Nakamura $^{e}$, Takahiro Oshima ${ }^{e}$, Toshiki Koike ${ }^{e}$, Shoichi Shibata ${ }^{f}$, Akitoshi Oshima ${ }^{f}$, \\ Hisanori Takamaru ${ }^{f}$, Hiroshi Kojima ${ }^{g}$, Harufumi Tsuchiya $^{h}$, Kyoko Watanabe $^{i}$, \\ Masayoshi Kozai ${ }^{j}$, Tatsumi Koi ${ }^{k}$ \\ E-mail: anzorena@geofisica.unam.mx
}

Solar neutron telescopes (SNT) were designed and installed on high mountains to study particle acceleration mechanisms in solar surface. Of these, SciBar cosmic ray telescope (SciCRT) is a brand new telescope installed on the top of the Sierra Negra volcano in eastern Mexico $\left(19^{\circ} \mathbf{N}\right.$, $97.3^{\circ} \mathbf{W}$ ) composed of roughly 15000 scintillator bars, capable of detecting solar particles with both high efficiency and energy resolution. SciCRT is also useful to study the anisotropy of galactic cosmic ray muons. The implementation of SciCRT as a cosmic ray telescope began on September 2013, with $5 / 8$ of the complete detector operative. After that, further improvement of the operating conditions on the place were made in order to maintain a stable data acquisition on the severe atmospheric conditions on high mountain (4600 m). In July 2015 we partially upgrade the DAQ system, installing a 10 times faster readout back-end electronics. The new system was installed only on $1 / 8$ of the detector and on the top and bottom layers (working as muon detectors and anti-counters for neutrons). The upgrade was motivated by the limited transfer rate of the original DAQ system, designed for an accelerator experiment. With this new system we expect an improvement on the neutron sensitivity of the SciCRT. In this paper we will detail the operation of the new system and analyze the data obtained to evaluate its performance. We will also explain the plans to continue the upgrading process, installing the new DAQ systems on more layers of the telescope and make estimates of the expected future performance of the SciCRT, evaluated through Monte Carlo simulations.

35th International Cosmic Ray Conference

10-20 July, 2017

Bexco, Busan, Korea

\footnotetext{
* Speaker.

${ }^{\dagger a}$ Instituto de Geofísica, Universidad Nacional Autónoma de México, Ciudad de México, 04510, México.

$\dagger^{\dagger}$ Institute for Space-Earth Environmental Research, Nagoya University, Furo-cho, Chikusa-ku, Nagoya 464-8601,

${ }^{\dagger c}$ Instituto de Ciencias Físicas, Universidad Nacional Autónoma de México, Cuernavaca, Morelos, 62210, México.

${ }^{\dagger}{ }^{d}$ SCiESMEX, Instituto de Geofísica, Unidad Michoacán, Universidad Nacional Autónoma de México, Michoacán Morelia, 58190, México.

${ }^{\dagger}$ Department of Physics, Shinshu University, Asahi, Matsumoto, 390-8621, Japan.
} Japan. 


\section{Introduction}

Solar neutrons are produced by the interaction of the accelerated ions with the solar atmosphere during solar flares. It is well know that neutrons carry important information about ion acceleration process: e. g. the starting time of ion acceleration, if acceleration process is instantaneous or gradual. Since neutrons have no electric charge, they arrive at the Earth unaffected by any magnetic fields along their path. However because neutrons have mass they suffer velocity dispersion, and as a consequence in order to establish the neutron emission profile at the Sun we need to measure both their energies and arrival times at Earth. Solar neutron telescopes (SNT) were designed and installed on high mountains with this purpose. So far around 10 solar neutron events have been observed, but those are not sufficient for clarifying the solar particle acceleration. This is one motivation behind the SciCRT experiment, to been able to construct a superior SNT with higher sensitivity for solar neutron observations and better energy resolution.

The SciCRT is also used as muon telescope, part of the Global muon detector network with the goal studying the directional anisotropy of galactic cosmic rays observed at the Earth.

\section{Description of the SciCRT experiment}

The SciCRT was installed on the top of Sierra Negra Volcano in Mexico $\left(19^{\circ} \mathbf{N}, 97.3^{\circ} \mathbf{W}\right)$, $4600 \mathrm{~m}$ above sea level [1]. This location is an excellent place to observe solar neutrons because its high altitude minimizes attenuation by the atmosphere. A picture showing SciCRT is on figure 1.

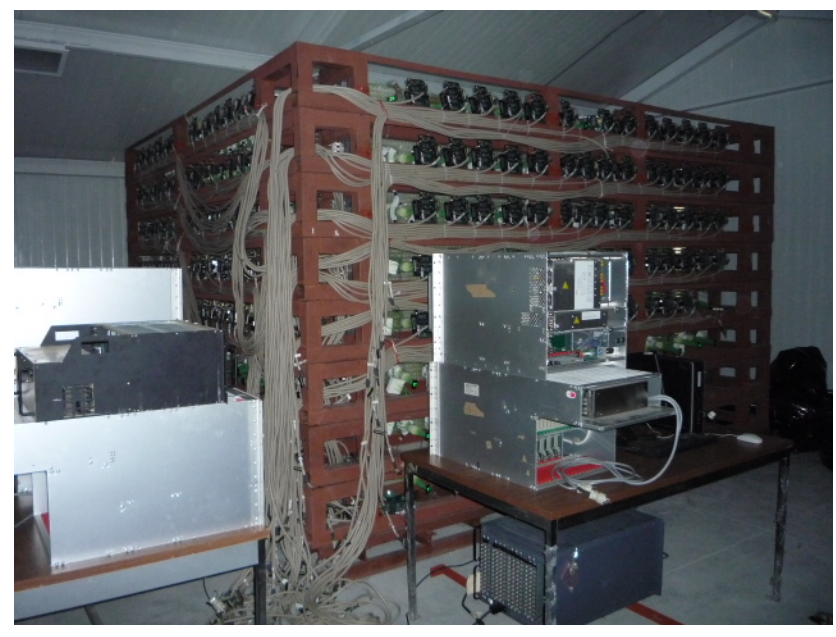

Figure 1: A picture of SciCRT installed on Sierra Negra volcano.

\footnotetext{
${ }^{f}$ College of Engineering, Chubu University, Kasugai 487-8501, Japan

${ }^{g}$ Faculty of Engineering, Aichi Institute of Technology, Toyota 470-0392, Japan

${ }^{h}$ Japan Atomic Energy Agency, 2-4 Shirakata Shirane, Tokai-mura, Naka-gun, Ibaraki 319-1195, Japan

${ }^{i}$ National Defense Academy of Japan, 1-10-20 Hashirimizu, Yokosuka, Kanagawa 239-8686, Japan

${ }^{j}$ Institute of Space and Astronautical Science, Japan Aerospace Exploration Agency, Sagamihara, Kanagawa 2525210, Japan.

${ }^{k}$ SLAC National Accelerator Laboratory, Menlo Park, CA 94025-7015, USA.
} 
The telescope uses the SciBar detector originally designed for neutrino oscillation experiment [2]. SciBar is constructed with 14848 scintillator bars, each of dimensions $1.3 \times 2.5 \times 300.0 \mathrm{~cm}^{3}$. The plastic scintillators allow us to record with precision the energy deposited along the path by recoil protons and identify the direction of incident neutrons. The bars are arranged in layers, each consisting of two orthogonal planes X and Y, with 116 bars on every plane. The are in total 64 layers of scintillator strips in the detector.

The scintillation light is collected by wavelength shifting fibers (WLS fibers) of $1.5 \mathrm{~mm}$ diameter inserted on the middle of every bar. The collected photons from 64 fibers are read by a 64 channel multi-anode photomultiplier tube (MAPMT) H8804 by Hamamatsu Photonics. The information from MAPMTs is read by a combination of front-end boards (FEBs) and back-end electronics, which were originally designed and used in the previous experiment [3]. One FEB has two application specific integrated circuits (ASICs), employed to multiplex pulse height information from the anodes of the MAPMT and make fast triggering signal (hit signal). The back-end board can readout 8 FEBs and is developed as a VME board. In this module, the analog signals from the FEBs are digitized (ADC data) and send to a DAQ server by a VME bus interface. With this system, a maximum transfer rate of $1 \mathrm{kHz}$ is achievable when reading only one BEB [4]. The trigger board (TRGB) collects the hit signals from FEBs and triggers other modules to capture cosmic rays events. Detection of muons is possible using four-fold coincidence from top and bottom layers as trigger. Neutral particles are identified when a track is registered in the middle of the detector without triggering any muon layer. Scaler data for neutral particles is also obtained, recording the number of hit signals on each MAPMT in anti-coincidence with the muon layers.

SciCRT has a large effective area, with a 10 times higher detection efficiency (for neutrons with $100 \mathrm{MeV}$ ) than the Solar neutron telescope previously installed on Sierra Negra [1]. SciCRT has also better energy resolution and particle identification capabilities.

\section{Current status of the SciCRT}

Because the electronic system was originally designed for an accelerator experiment, the specific requirements depart from our goals as a Solar neutron/cosmic ray experiment. Considering the maximum transfer rate of the DAQ system is limited to (under the best condition) $1 \mathrm{kHz}$ with the VME bus interface and that the rate of background cosmic ray neutrons at Sierra Negra is superior to $2 \mathrm{kHz}$, the overall sensitivity of SciCRT to solar neutrons is considerably affected by the long dead time. Furthermore, the VME interface induces noise on the transmission line of the hit signals from the BEB when ADC data are transfer, and therefore affecting scaler data acquisition.

On the other hand, the severe environmental conditions ( $4600 \mathrm{~m}$ above sea level) at which the DAQ system needs to operate need to be taken into account on the design of the system.

Motivated by this, we designed and installed an improved version of the DAQ system on July 2015 on the muon layers and 1/8 of the detector. The new DAQ system uses SiTCP [5] (a hardware network processor implemented on FPGA) based BEBs to read information from 4 FEBs to one DAQ server using TCP/IP protocol. A full description of the system and its basic performance is found elsewhere [4]. After testing the operation of the new BEB on the SciCRT we conclude that this new system has the capability to obtain neutron ADC data about 10 times faster than the old 
BEB. We also determined that the noise problem originated in the previous BEB was eliminated in the new design, and hence the acquisition of scaler data for neutral particles is now possible.

Regarding the operational condition of the electronics, we installed an uninterruptible power supply specially designed for the SciCRT. With this system, a battery bank is continuously charging and if there is an electrical power cut, the DAQ system is capable of maintaining operation until electrical power recovered. Besides, the new DAQ system is equipped with powerful air circulator which enables efficient heat dissipation in a low pressure environment.

After this measures and an improvement on the control software of the detector (scheduling of data taking process and compression), the overall stability of the event rate on SciCRT increase. A example of this is shown of figure 2. In this figure we shown the detected event rate by the muon layers during the May 2017. The mean counting rate is $446 \mathrm{~Hz}$ with an overall stability of $1 \%$. It is important to notice that this event rate is considering only the top and bottom layers detecting muons, and is different from the event rate for background neutrons stated before. This result is consistent with the ones presented in $[1,4]$.

To further prove our point, we analyzed the muon ADC data during the same time period. In this case we obtained a mean ADC distribution per FEB, and there are 8 FEBs responsable for muon data. One example of this distributions is shown in figure 3. Considering that each FEB has 64 channels, and each channel has different gain, we first need to normalize the data in order to be able to compare between different channels. After this we are able to obtain the global ADC distribution per front-end board shown figure 3. A detailed description of this distribution is on the legend of the figure.

For separating the pedestal distribution from the cosmic ray signal, we fit a gaussian distribution to the pedestal peak and a Landau distribution to the muon signal. With this approach the peak for the cosmic ray signal is $285 \mathrm{ADC}$, which is the value set in the initial calibration of the SciCRT [6]. All FEBs on the muon layers have similar behavior during the period of analysis, which demonstrates the stability of the ADC data on this section of the detector.

In the case of the neutral particle data, a similar analysis is far more complex and still a work in progress, mainly due to the larger data volume. The $1 / 8$ of the detector (called super block) in which the DAQ system is installed is composed of 28 FEBs, with a total number of 1792 different channels of data.

Nevertheless, to ensure the operation of the neutral channels we proceed with a different type of analysis. In this we search for registered events that cross more than 4 layers of scintillator bars in the $\mathrm{X}$ or $\mathrm{Y}$ side of the detector. Because the muon layers serve as anti-coincidence signal for these super block (SB), all the registered tracks start somewhere in the middle of the detector (above or in the SB in question). A example showing a neutron like track is on figure 4. Since there is only one SB working at the time, we could no see the starting and ending point of the track. Neutron induced events have the distinguish feature of large energy deposit at the end of the track produced by recoil protons.

With the criteria explained above, we can search for recorded events with similar features. To reduce the number of data to process we choose three random days during May 2017. After applying the selection criteria, only around the $12 \%$ of the events are candidates. If we increase the criteria to select events crossing all 8 layers of the SB, only $1.0 \%$ are candidates. 


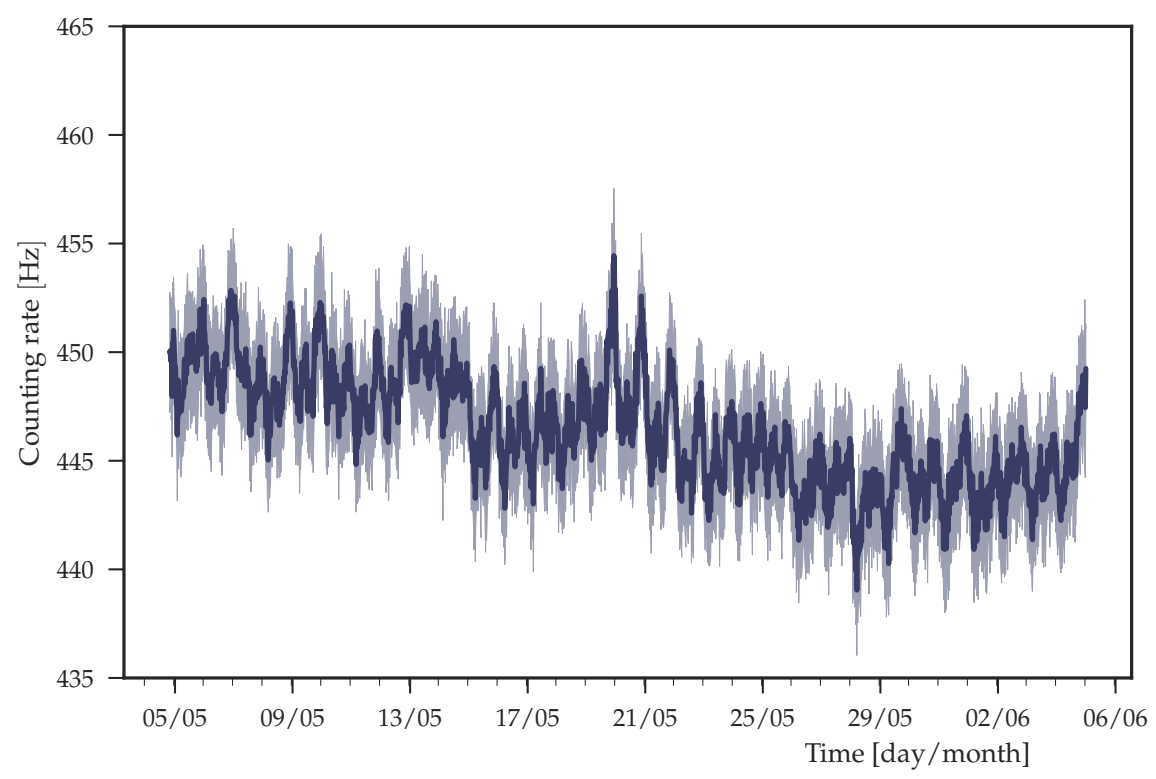

Figure 2: Muon ADC data event rate during the month of May. The blue thick line represents the average counting rate per hour. The light shaded areas corresponds to the $\pm \sigma$ interval.

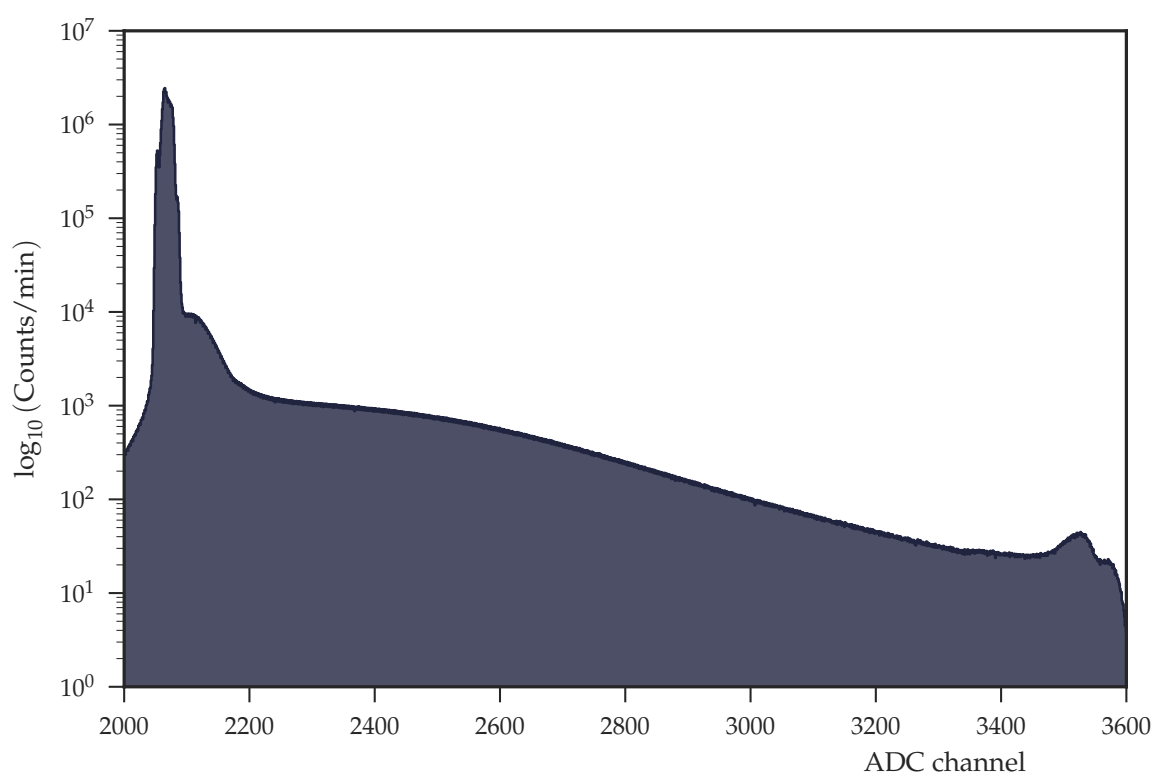

Figure 3: Average ADC distribution from one FEB during the month of May. Low ADC values (in the range from $2000-\approx 2100$ ) correspond to pedestal data while higher values correspond to cosmic ray muons signal. The large peak in the final part of the distribution (around 3400 ADC) is caused by the effect of saturation from the FEBs. 
From the selected candidates, we wanted to test if the majority corresponds to proton recoil tracks. To be able to compare between each track we must first normalize each track by the maximum ADC value recorded in it and consider the position of pedestal data on each channel. The second step is to obtain the energy loss per unit length (per layer) for all the candidates. Figure 5 shows the result of this analysis on each side of the detector using the criteria of 8 layers. As it is expected for proton recoil tracks, the energy deposition increases as the particle travels through the detector. After reaching a maximum, the energy deposition suddenly decreases. A similar result is also obtained using the selection criteria of 4 layers. With this result we can conclude that the majority of the selected tracks are neutron-like, and that in principle, the new data acquisition system is capable of obtaining reliable ADC neutron data.

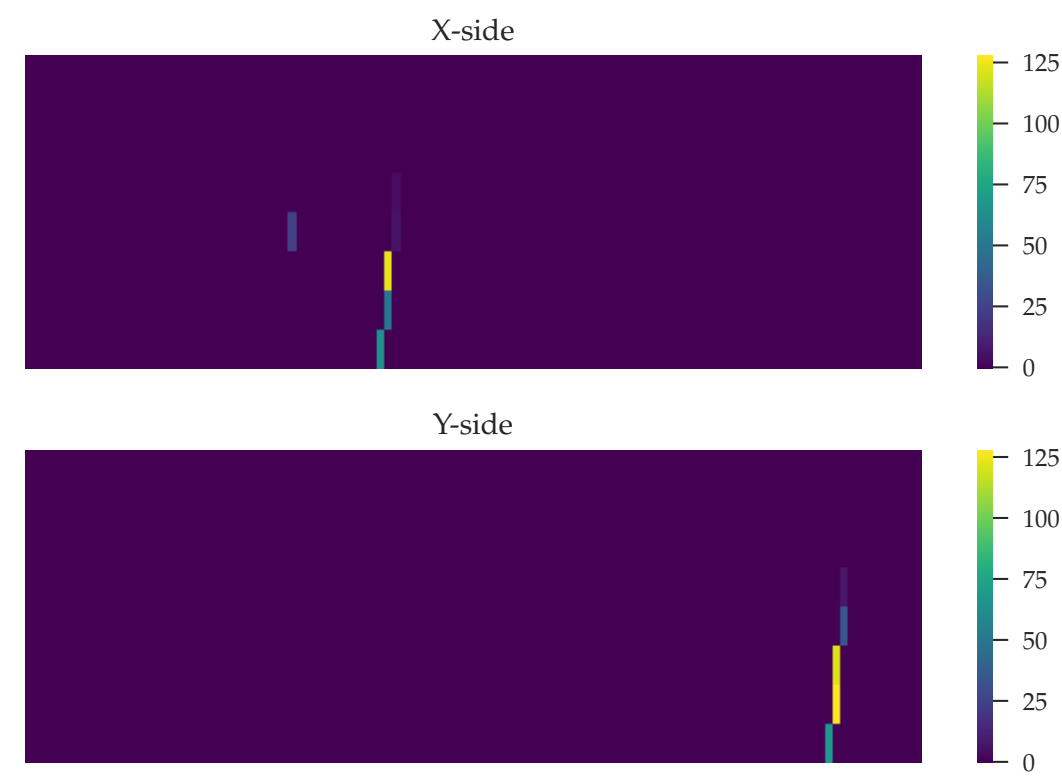

Figure 4: Example data track showing features similar to the interaction of a low energy neutron.

\section{Future expected performance}

We may evaluate the response of SciCRT to a solar neutron event by means of Monte Carlo (MC) simulation and compare it with the results obtained with the other SNT in Mexico. For doing so, we will consider the 7 September 2005 solar neutron event [7]. This event was detected by the SNT in Mexico ( $>30 \mathrm{MeV}$ neutral particle channel) with a significance of $17 \sigma$. The peaking time of the hard X-rays detected by Geotail satellite was 17:36:40 UT.

Assuming the solar neutron flux of this event, we may simulate how significant the SciCRT's signals would be when detecting this event. For the MC simulation we consider the following parameters [1]: the energy spectrum of neutrons at the sun is represented by a power law as $6.1 \times 10^{27}(E / \mathrm{MeV})^{-3.8} \mathrm{MeV}^{-1} \mathrm{sr}^{-1}$, neutrons are emitted impulsively at the Sun, the solar zenith angle of the Sun is fixed to $17.5^{\circ}$. The propagation of solar neutrons through the atmosphere of the Earth is calculated using the Shibata model [8]. 

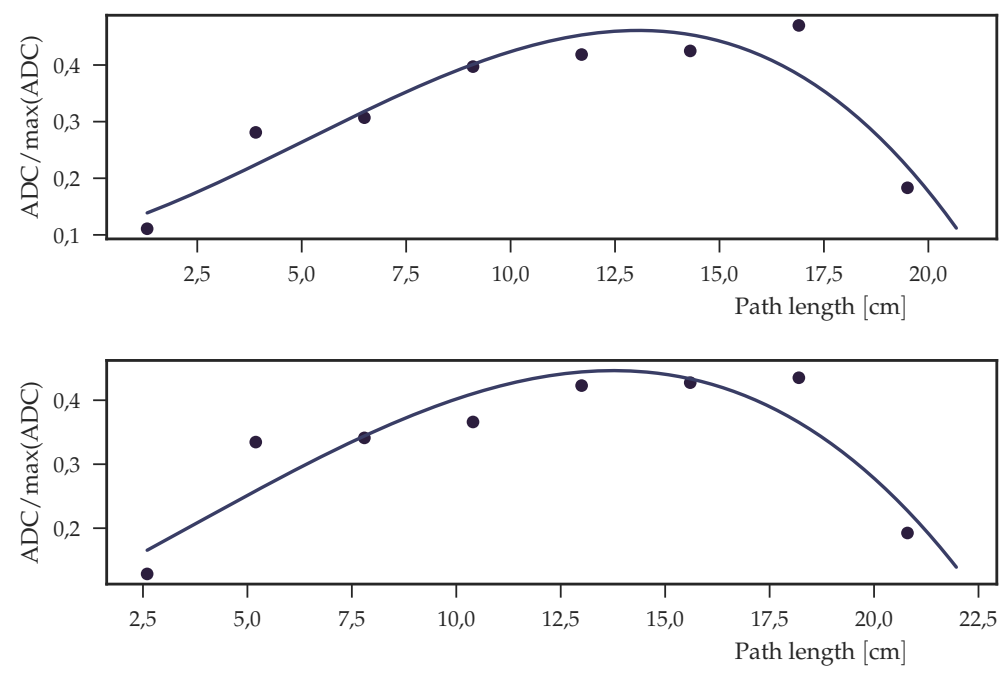

Figure 5: Mean ADC value as a function of path length for events that cross 8 layers of bars or more.

According to this calculation, the neutron scaler data using 4/8 of the SciCRT with the old DAQ system would have a $39 \sigma$ significance during the event. This means SciCRT will be 2.3 times more sensitive to solar neutron events than the Mexico SNT. Yet, this sensitivity is still significantly limited by the maximum transfer speed of the original modules. Considering we install the improved DAQ modules described before in $4 \mathrm{SBs}$, the significance is enhanced up to $59 \sigma$, that is 1.5 times more sensible. Figure 6 [4] shows the results of the simulation with the expected time profiles of neutron scaler data during the event in the case of using 4 SBs in comparison with data obtained by Mexico SNT during this event. Concerning ADC data, the installation of new DAQ modules will increase the significance 3 times and allow to determine a precise energy spectrum.

In the near future we plan to make further improvements on the detector, all of which will have a impact on the performance of the SciCRT. In the next brief list we summarize the currently undergoing tasks: 1 ) implementation of a selective trigger mode which enables the suppression of pedestal data, 2 ) further improvement of the throughput rate of the BEB, with the installation of more DAQ servers to control the data acquisition and 3 ) development of new low-cost front-end electronics to enable the full installation of the SciCRT.

\section{Summary}

The SciCRT is an improved solar neutron telescope, which began operations on September 2013 using the original DAQ system developed for an accelerator experiment. Because the transfer rate limitations of these modules, we developed back-end electronics based on the fast TCP/IP protocol. We successfully installed the new DAQ system on Sierra Negra on July 2015. Aside from the almost 10 times better transfer rate obtained with the new system, the analysis of the most recent data shows an improvement on the overall stability of the data. Furthermore, throughout detailed $\mathrm{MC}$ simulations we have been able to determine the future expected performance of SciCRT during a solar neutron event. 


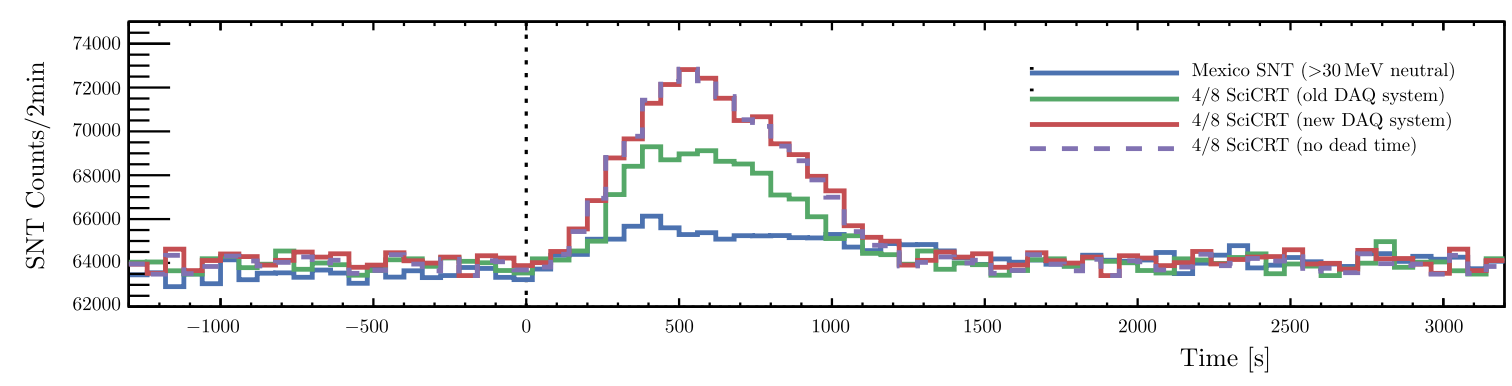

Figure 6: Simulated time profiles of neutron scaler data from the SciCRT assuming the solar neutron event of 7 September 2005. The dot dashed line on $0 \mathrm{~s}$ corresponds to the peak time of hard X-rays observed by satellite. The blue is data obtained by Mexico SNT, green line is SciCRT's time profile using original DAQ system, while red line is time profile with new DAQ system installed. The purple dotted line is ideal situation with no dead time. All data are normalized by background of Mexico SNT data. Figure taken from [4].

\section{Acknowledgments}

We want to thank the SciBar and SciBooNe experiments for allowing us to use the SciBar as a cosmic ray detector. We are also grateful to the National Institute of Astrophysics, Optics and Electronics (INAOE) for allowing us to continue the experiment at Sierra Negra. We also thank the help on the development from the Open-It consortium. Finally we want to thank Mexican Physics Society and local organizing committee of the 35th ICRC for the funds to attend conference. This work is partially supported by UNAM-PAPIIT-IN104115 and CONACyT-180727T. Grants from Scientific Research(B) 22340054, Scientific Research(C) 23540348 and Fellows 2434-0054 from JSPS are also acknowledged. This work is also partially supported by the joint research program of the Solar-Terrestrial Environment Laboratory (STEL), Nagoya University, Japan.

\section{References}

[1] Y. Nagai et al., First cosmic-ray measurements by the SciCRT solar neutron experiment in Mexico, Astroparticle physics 59 (2014) 39-46.

[2] K. Nitta et al., The K2K SciBar detector, Nuclear Instruments and Methods in Physics Research A 535 (2004) 147-151.

[3] M. Yoshida et al., Development of the readout system for the K2K SciBar detector, IEEE transactions on nuclear science 51:6 (2004) 3043-3046.

[4] Y. Sasai et al., A faster and more reliable data acquisition system for the full performance of the SciCRT, Nuclear Instruments and Methods in Physics Research A 857 (2017) 50-57.

[5] T. Uchida, Hardware-Based TCP Processor for Gigabit Ethernet, IEEE transactions on nuclear science $\mathbf{5 5}$ (2008) .

[6] Nagai, Y., Observation of cosmic rays by the new solar neutron telescope, ph.d. thesis, Nagoya University, 2014.

[7] T. Sako et al., Long-lived solar neutron emission in comparison with electron-produced radiation in the 2005 september 7 solar flare, Astrophysical Journal 651 (2006) L67-L72.

[8] S. Shibata, Propagation of solar neutrons through the atmosphere of the Earth, Journal of geophysical research 99 (1994) 6651-6665. 